\title{
Establishment of a murine pancreatic cancer pain model and microarray analysis of pain-associated genes in the spinal cord dorsal horn
}

\author{
LIQIN WANG ${ }^{1,2}$, HUIHONG XU ${ }^{1}$, YANHU GE $^{3}$, HAI ZHU ${ }^{4}$, DAWEI YU ${ }^{5}$, WEIFENG YU ${ }^{1}$ and ZHIJIE LU ${ }^{1}$ \\ ${ }^{1}$ Department of Anesthesiology and Intensive Care Unit, Eastern Hepatobiliary Surgery Hospital, \\ Second Military Medical University, Shanghai 200438; ${ }^{2}$ Department of Anesthesiology, 85th Hospital of CPLA, \\ Shanghai 200052; ${ }^{3}$ Department of Anesthesiology, 309th Hospital of CPLA, Beijing 100091; ${ }^{4}$ Department of \\ Anesthesiology, Maternal and Child Health Hospital of Putuo, Shanghai 200061; ${ }^{5}$ Department of \\ Anesthesiology, 101th Hospital of CPLA, Wuxi, Jiangsu 214044, P.R. China
}

Received October 8, 2016; Accepted March 10, 2017

DOI: $10.3892 / \mathrm{mmr} .2017 .7173$

\begin{abstract}
There is emerging evidence on the mechanisms of pancreatic cancer pain. Following the establishment of an orthotropic transplantation model of pancreatic cancer, microarray analysis was performed to identify changes in the expression levels of pain-associated genes in the spinal cord. A mouse model of pancreatic cancer-induced pain was established by implanting SW 1990 cells into the pancreases of female BALB/c-nu mice. The survival rate and body weight were measured following orthotropic transplantation. Gross anatomical techniques and hematoxylin and eosin staining were used to analyze the pancreatic tumor tissue. Multiple behavioral tests were also performed to assess pain-associated responses. Additionally, using samples from mice with or without observable pain, microarray analysis was performed to determine the gene expression profiles in the spinal cord dorsal horn. The survival rate of mice with pancreatic cancer was high during the initial 3 weeks post-surgery, although the body weight decreased progressively. Gross anatomical techniques demonstrated that the tumor size increased significantly following the surgery, and this result was confirmed by solid tumor masses in the pancreatic tissues of the mouse model. Observable pain behavioral responses were also examined in the pancreatic cancer model by measuring the mechanical threshold of the abdominal skin, hunching behavior and visceromotor responses. The profiles of 10 pain
\end{abstract}

Correspondence to: Professor Weifeng Yu or Professor Zhijie Lu, Department of Anesthesiology and Intensive Care Unit, Eastern Hepatobiliary Surgery Hospital, Second Military Medical University, 225 Changhai Road, Shanghai 200438, P.R. China

E-mail: ywf808@yeah.net

E-mail: 1zjwxyz@163.com

Key words: pancreatic cancer pain, mouse model, pain behavior, spinal cord dorsal horn, gene express profile specific-associated genes in the spinal cord dorsal horn that accurately reflect the molecular pathological progression of disease were also identified. In conclusion, the present study has developed a novel animal model of pancreatic cancer pain in $\mathrm{BALB} / \mathrm{c}-\mathrm{nu}$ mice that resembles human pancreatic cancer pain, and the expression of pain-associated genes in the spinal cord dorsal horn has been profiled. The results of the present study may further the understanding of the molecular mechanisms that mediate pancreatic cancer pain.

\section{Introduction}

Over 10 million people develop cancer every year and this number is likely to increase to 15 million by 2020 (1). Previous studies have demonstrated that the prevalence of pain in patients with cancer is high, between $50-90 \%(2-4)$. The severe pain associated with cancer profoundly impairs the quality of life and threatens the survival rate of patients. Despite the availability of various therapeutic and treatment strategies, there remains no effective analgesic treatment, as the mechanisms involved in the cancer-associated pain are poorly understood (5-7).

Pancreatic cancer causes one of the severest forms of cancer-associated pain. To investigate human pancreatic cancer-associated pain, animal models are required to provide insights into the mechanisms that drive cancer pain and to develop improved treatment strategies for tumor-induced pain. Animal models of pancreatic cancer have been developed in rodents. Tevethia et al (8) reported that transgenic mice expressing an N-terminal T-antigen segment (T1-127) and small $\mathrm{T}$ antigen exhibited acinar cell dysplasia at birth and progressed to neoplasia. Loukopoulos et al (9) established a series of orthotropic models of human pancreatic adenocarcinoma in severe combined immunodeficiency mice using pancreatic cancer cell lines and primary tumors. However, the model focused on the pathobiology of pancreatic cancer, and few studies have investigated the associated pain.

The present study established a clinically relevant orthotropic transplantation model of pancreatic cancer pain in mice. 
This model yielded a higher success rate and lower mortality compared with previous models. The study demonstrated that the development of tumors markedly decreased the abdominal withdrawal threshold and resulted in behavior associated with severe pain, which mimicked human pancreatic cancer pain.

Following monitoring of the mechanical pain behavior of pancreatic cancer mice, the spinal cord dorsal horn tissues were harvested from the mice with or without observable pain and from the sham-operated mice. The samples were used to examine the gene expression profiles in the different groups, determining the pain-associated changes in gene expression during pancreatic cancer, and also to improve the understanding of the molecular mechanisms of cancer pain.

\section{Materials and methods}

Animals. Adult female BALB/c-nu mice were obtained from Sino-British SIPPR/BK Laboratory Animal Ltd. (Shanghai, China) and housed under a 12-h light/dark cycle at $24 \pm 0.5^{\circ} \mathrm{C}$ with food and water available ad libitum. All experiments were performed with the approval of the Animal Care and Use Committee of the Second Military Medical University (Shanghai, China), and followed the policies issued by the International Association for the Study of Pain on the use of laboratory animals.

Cell culture. The pancreatic cancer cell line (SW 1990) was obtained from the Department of Gastroenterology, Changhai Hospital of Second Military Medical University (Shanghai China). SW 1990 cells were cultured in RPMI-1640 medium, supplemented with $10 \%$ fetal bovine serum (both from Gibco; Thermo Fisher Scientific, Inc., Waltham, MA, USA). The medium was replaced every 3 days and the cells were cultured in humidified air containing $5 \% \mathrm{CO}_{2}$.

Mouse model of pancreatic cancer. Female BALB/c-nu mice $(20 \pm 0.69 \mathrm{~g}, \mathrm{n}=120 ; 5$-week-old) were acclimatized and maintained in a specific pathogen-free environment. Mice were anesthetized with isoflurane in $\mathrm{O}_{2}$ (2\% induction, $1.5 \%$ maintenance), the abdomen opened by an incision in the left lumbar region and the pancreatic tail and the spleen were exteriorized. SW 1990 cells $\left(\sim 3 \times 10^{6}\right.$ in a $20 \mu 1$ suspension) were slowly injected into the pancreas and formed a $2-3 \mathrm{~mm}$ vesicle using an inoculator fitted with a 26 -gauge needle. The injection site was sealed by COMPONT (synthetic cyanoacrylic glue; Beijing Compont Medical Devices, Beijing, China). Subsequently, the pancreas and the spleen were relocated into the abdominal cavity, and the incision was closed using silk thread. Sham groups were injected with the same volume of medium as a control. All surgery was performed in an ultra clean cabinet.

Survival rate analysis, body weight, gross anatomy and histology. The mice were observed daily for general well-being and twice weekly to monitor tumor formation by palpation until euthanasia criteria were reached (up to 30 days post-surgery) Euthanasia criteria included either $30 \%$ weight loss or extreme weakness, or inability to obtain food/water.

Survival rate (SW 1990, $\mathrm{n}=40$; sham, $\mathrm{n}=40$ ) and body weight following SW 1990 xenografting or sham surgery were analyzed. The survival rates of mice that received SW 1990 xenografts were compared with mice that received sham surgery.

Mice were sacrificed using isoflurane at 1,2,3 and 4 weeks post-xenograft or sham surgery (SW 1990, $n=10$; sham, $\mathrm{n}=10$ ). Following euthanasia, a post-mortem examination was performed on all animals and the pancreas the spleen and duodenum were dissected from the peritoneal viscera. Images of the pancreas were captured with a digital camera (Canon IXUS 870 IS; Canon, Inc., Tokyo, Japan). Subsequently, the chests of the mice were opened and the aorta perfused with saline, followed by cold fixative (4\% formaldehyde in $0.1 \mathrm{M}$ phosphate buffer at $\mathrm{pH}$ 7.4). The pancreas was dissected from the peritoneal viscera, post-fixed overnight and then placed in $30 \%$ sucrose until equilibration.

The pancreases were embedded in paraffin wax and sectioned into $8 \mu \mathrm{m}$ slices. The slices were dewaxed with dimethylbenzene and ethanol solutions. Then, the sections were stained in hematoxylin and eosin (H\&E). Sections were observed with a Nikon optical microscope (Nikon Corporation, Tokyo, Japan) at x400 magnification, and images were captured with a charge-coupled device (CCD) camera.

Pain behavioral testing. Mice were examined from 9:00 to 11:00 in the morning for pancreatic cancer-induced pain using behavioral testing (SW 1990, $\mathrm{n}=10$; sham, $\mathrm{n}=10$ ). The observer was blinded to the experimental group of the mice.

The behavioral testing was performed weekly from day 1 of SW 1990 xenografting or sham surgery. Initial behavioral testing of each mouse was performed prior to the surgery.

Abdominal withdrawal threshold. The withdrawal thresholds to mechanical stimuli were used as an indicator of mechanical sensitivity of the abdominal skin. The mechanical thresholds were measured using electronic von Frey apparatus (IITC, Woodland Hills, CA, USA). The electronic von Frey apparatus employs a non-flexible filament. Mice were placed in a plastic chamber $(8.0 \times 8.5 \times 20 \mathrm{~cm})$ on top of a mesh screen platform and habituated for $10 \mathrm{~min}$. The stimulus was applied to the skin of the epigastric region with increasing force. An abdominal withdrawal response or whole-body withdrawal during stimulation was considered as a positive response. Values obtained from the apparatus were recorded as the withdrawal thresholds. The mean thresholds were calculated from five consecutive trials (each performed every $15 \mathrm{~min}$ ).

Hunching. Observed hunching behavior was scored from 0 to 4 as follows: 0 , exhibited exploratory behavior and lack of a rounded-back posture; 1 , exhibited exploratory behavior and mild rounded-back posture; 2, exhibited slightly reduced exploratory behavior, severe rounded-back posture and intermittent abdominal contractions; 3 , exhibited markedly reduced exploratory behavior, severe rounded-back posture and intermittent abdominal contractions; and 4, exhibited little or no exploratory behavior, severe rounded-back posture and intermittent abdominal contractions. Mice were placed in the center of an open arena with the floor covered with clean non-woven fabric and observed over a $300 \mathrm{sec}$ period. The hunching score was based on the accumulated time (sec) engaged in the hunching behavior multiplied by the scoring 


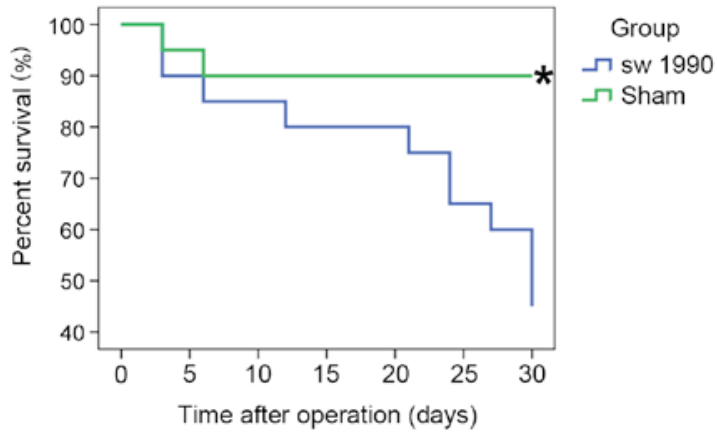

Figure 1. Kaplan-Meier curve demonstrating survival rates following SW 1990 xenografts or sham surgery. ${ }^{*} \mathrm{P}=0.004$ vs. SW 1990 mice, log-rank test. SW 1990, SW 1990 cell xenografted mice $(n=40)$; sham, sham-operated mice $(n=40)$.

factor associated with that behavior's observed degree of impairment (10).

Visceromotor responses (VMRs). On day 14 post-surgery mice of the SW 1990 and sham groups (SW 1990, n=5; sham, n=5) were anesthetized with isoflurane. An incision was made in the skin of the epigastric region and two silver wires were implanted in the rectus abdominis. The wires were implanted subcutaneously and exteriorized at the back of the neck. The mice were subsequently housed one per cage for 7 days.

Mice were placed individually in the center of an open field arena and the implanted wires were connected to a preamplifier (P5 Series; Grass Technologies, Warwick, RI, USA), electrical activity from the rectus abdominis was relayed to the Spike2 data acquisition program (version 7 for Windows; Cambridge Electronic Design, Cambridge, UK). The processed signal data designated VMRs were exported to Igor Pro software version 6.0 (WaveMetrics, Lake Oswego, OR, USA) for analysis $(11,12)$, and subtracted from background readings. To normalize VMRs, all VMRs from SW 1990 xenografted mice and sham-operated mice were divided by VMRs from normal mice. For all testing, the mice were habituated for $10 \mathrm{~min}$, and then VMRs were recorded for $30 \mathrm{~min}$. VMRs were recorded 5 times for each mouse, and the mean VMRs were calculated.

Microarray analysis. As the pancreases were innervated by nerves from T8-12 in the spinal cord, total RNA was extracted from spinal cord dorsal horn tissues using the RNeasy Mini kit (Qiagen, Inc., Valencia, CA, USA). The mice were divided into three groups according to hunching score at day 14 post-surgery as follows: Mice with severe cancer pain $(\mathrm{SP} \geq 3)$, mice with minor cancer pain hypersensitivity $(\mathrm{MP} \leq 1)$ and sham mice as a control. The RNA concentration and purity were measured by optical density at 260/280 ( $\geq 1.8)$ and 260/230 ( $\geq 1.5)$, respectively, and the yield and quality were assessed using an Agilent 2100 Bioanalyzer (Agilent Technologies, Santa Clara, CA, USA). Fluorescent antisense (a)RNA probes were prepared from $1 \mu \mathrm{g}$ total RNA samples using a OneArray ${ }^{\circledR}$ Amino Allyl aRNA Amplification kit (Phalanx Biotech Group, Hsinchu, Taiwan) and Cy5 dyes (Amersham Pharmacia Biotech, Inc., Piscataway, NJ, USA). Fluorescent probes were hybridized to the Mouse Whole Genome OneArray ${ }^{\circledR}$ with Phalanx hybridization buffer using Phalanx hybridization system (Phalanx Biotech Group). Following $16 \mathrm{~h}$ hybridization at $50^{\circ} \mathrm{C}$, non-specific binding targets were washed away by 3 washing steps with $2 \mathrm{X}$ saline-sodium citrate (SSC) containing $0.2 \%$ sodium dodecyl sulfate, $2 \mathrm{X}$ SSC and $0.2 \mathrm{X}$ SSC solution. Finally the slides were dried by centrifugation and scanned using an Axon 4000B scanner (Molecular Devices, Sunnyvale, CA, USA). The intensities of each probe were measured using GenePix version 4.1 software (Molecular Devices). The raw intensity of each spot was submitted into the Rosetta Resolver ${ }^{\circledR}$ system (Rosetta Biosoftware, Seattle, WA, USA) to process data analysis. Probes that passed the threshold criteria were normalized using a 50\% median scaling normalization method. The technical repeat data were analyzed using Pearson's correlation coefficient calculation to determine the reproducibility (R-value $>0.975$ ). Normalized spot intensities were transformed to gene expression $\log _{2}$ ratios by comparing the control and treatment groups. The probes with $\log _{2}$ ratio $\geq 1$ or $\log _{2}$ ratio $\geq-1$ and $\mathrm{P}<0.05$ were defined as differentially expressed genes, and used in further pathway enrichment analysis.

Statistical analysis. Data are presented as the mean \pm standard error and analyzed using the SPSS version 13 (SPSS, Inc., Chicago, IL, USA). For analysis of body weight, mechanical threshold and hunching score, two-way repeated analysis of variance was used, followed by a post-hoc least significant difference (LSD) test. For survival analysis, Kaplan-Meier survival analysis was performed using log-rank testing. VMR results were analyzed by one-way analysis of variance. $\mathrm{P}<0.05$ was considered to indicate a statistically significant difference.

\section{Results}

Survival rate analysis in SW 1990 xenografted mice. Of the mice, 22 out of 40 succumbed within 30 days post-SW 1990 xenografting. By contrast, only 4 out of 40 mice succumbed following the sham surgery $(\mathrm{P}=0.004$, log-rank test; Fig. 1). Notably, all the 4 sham mice succumbed within the first week post-surgery and 6 mice succumbed within the first week post-SW 1990 xenografting, suggesting that the surgical trauma was an important factor in the mouse mortality during the first week post-surgery. In the sham surgery group, no mice succumbed after day 8 post-surgery, whereas 16 SW 1990 xenograft mice succumbed between day 8 to 30, and 12 SW 1990 xenograft mice succumbed after day 21 post-surgery. The survival rate of SW 1990 xenograft mice was high in the first 3 weeks, then declined significantly during the week 4 post-surgery, but remained $>50 \%$ until day 29 (Fig. 1).

Body weight analysis in SW 1990 xenografted mice. During the first week post-surgery, the body weight of mice in the SW 1990 group decreased notably, whereas it was only slightly decreased in the sham surgery group. The present study therefore concluded that surgical trauma and progression of cancer contributed to weight loss. Between days 8 and 28 post-surgery, the body weights of sham-operated mice increased gradually, whereas the body weights of SW 1990 xenograft mice were markedly reduced. The mean body weight of mice was $14.58 \mathrm{~g}$ on day 28 post-surgery, and SW 1990 xenografting resulted 


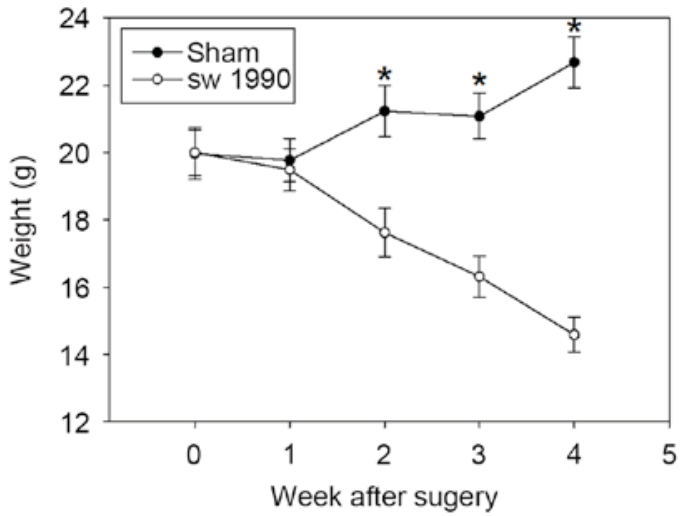

Figure 2. Weight analysis of SW $1990(\mathrm{n}=10)$ and sham-operated $(\mathrm{n}=10)$ mice. During week 1 post-surgery, the body weight of SW 1990 mice decreased significantly, and that of sham surgery mice decreased slightly. Between days 8 and 28 post-surgery, the body weight of sham-operated mice increased gradually; by contrast, the body weight of SW 1990 xenografted mice markedly decreased. ${ }^{*} \mathrm{P}=0.003$ vs. SW 1990 mice, post-hoc least significant difference test. SW 1990, SW 1990 cell xenografted mice; sham, sham-operated mice.
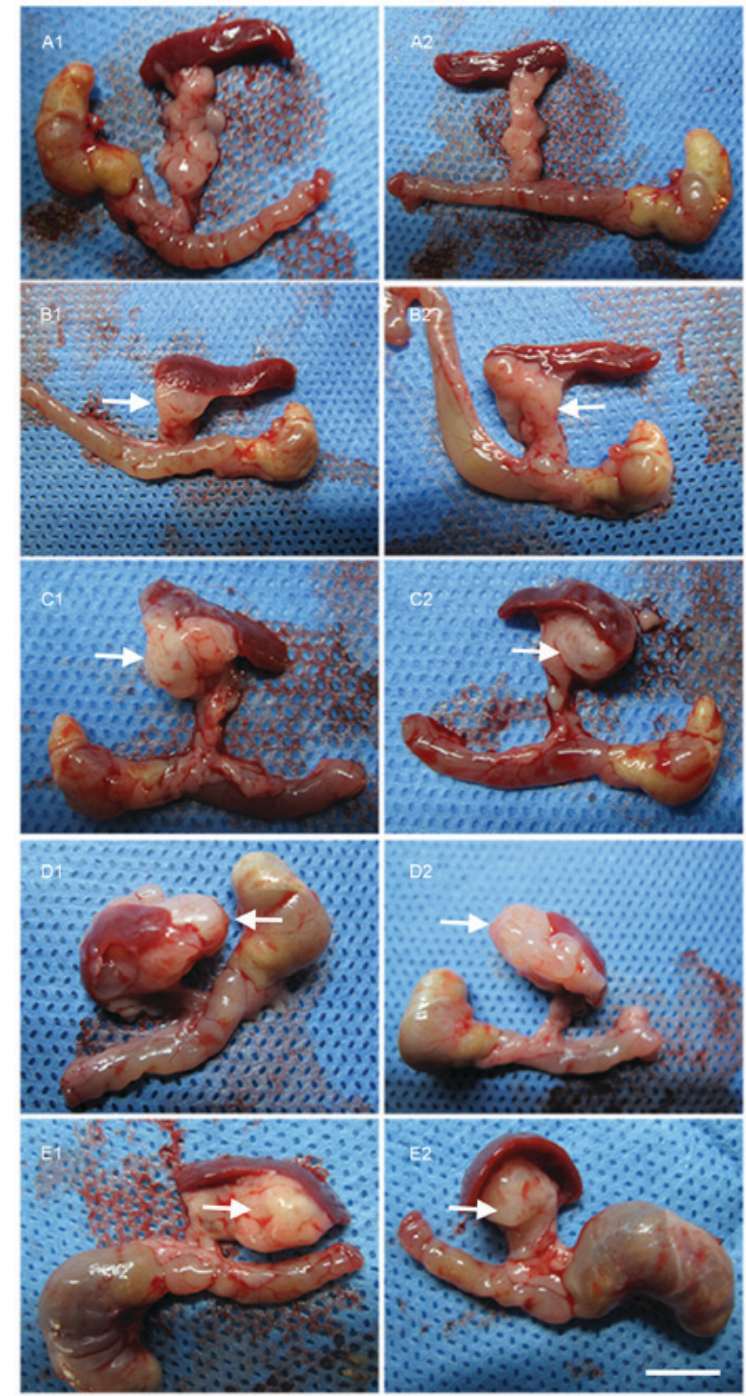

Figure 3. Gross anatomy in SW 1990 xenografted mice. (A1 and A2) Images of the anterior and posterior surface of normal pancreatic tissue. (B1-E2) Images of pancreatic tissue at weeks 1, 2, 3 and 4 post-SW 1990 xenografting. The tumor (arrows) volume increased continually over time following SW 1990 xenografting. Scale bar, $10 \mathrm{~mm}$.

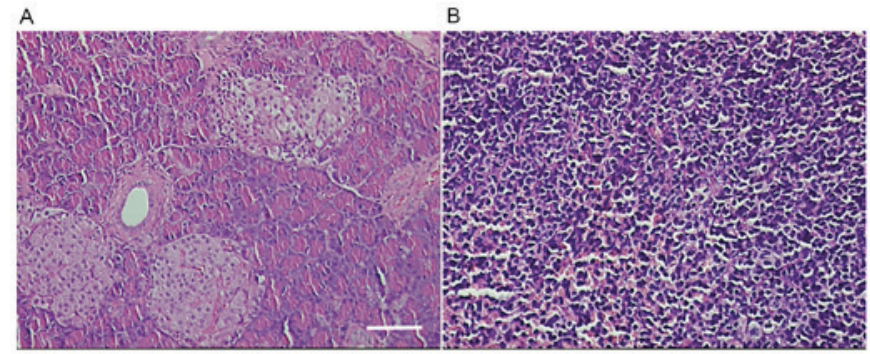

Figure 4. Hematoxylin and eosin staining of pancreatic tissue in mice 4 weeks post-SW 1990 xenografted and normal BALB/c-nu mice. (A) Normal pancreatic tissue. (B) Pancreatic tissue of SW 1990 xenografted mice. Fewer differentiated acinar cell carcinomas and larger solid tumor masses were observed in the SW 1990 group. Scale bar, $100 \mu \mathrm{m}$.

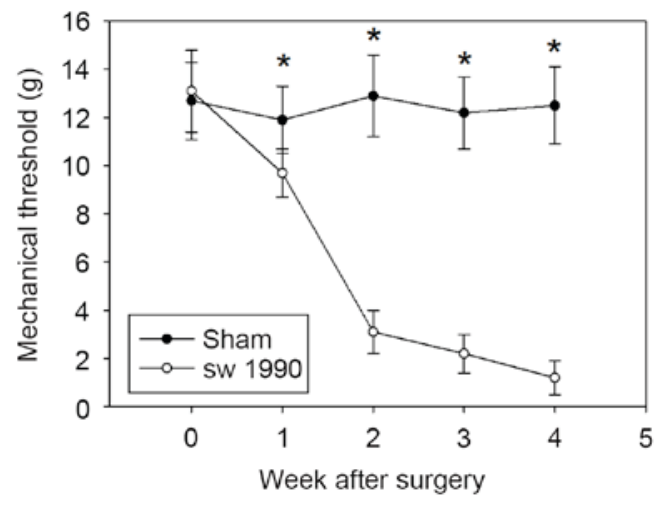

Figure 5. SW 1990 xenografting decreases the mechanical threshold of abdominal skin in mice ( $\mathrm{n}=10$ for each group). The mechanical thresholds of the SW 1990 xenografted mice remained at the same levels as the sham-operated mice at day 0 post-surgery. ${ }^{*} \mathrm{P}<0.001$ vs. SW 1990 mice by post-hoc least significant difference test. SW 1990, SW 1990 cell xenografted mice; Sham, sham-operated mice.

in a $27.1 \%$ reduction in the body weight of mice over 4 weeks $(\mathrm{P}=0.003$, post hoc LSD test; Fig. 2).

Pancreatic gross anatomy and histology in SW 1990 xenografted mice. To examine the development of pancreatic cancer in the mice, gross anatomy was performed to compare the normal pancreatic tissue (Fig. 3A1 and A2) and pancreatic tissue from weeks 1, 2,3 and 4 post-sham surgery and SW 1990 xenografting. Images of the anterior and posterior surface of the pancreases were captured for analysis. At week 1 post-SW 1990 xenografting, solid pancreatic tumors were observed in situ (Fig. 3B1 and B2). The tumor diameter increased in increments from $\sim 3$ to $10 \mathrm{~mm}$ following SW 1990 xenografting (Fig. 3B1-E2).

$\mathrm{H} \& \mathrm{E}$ staining was performed to compare pancreatic tissues from SW 1990 xenografted mice and normal BALB/c-nu mice at week 4 post-surgery. In the normal mice, histological analysis demonstrated that endocrine cells were well arranged, and normal islets were observed. The endocrine cells and islets were localized around capillaries for the delivery of secreted hormones (Fig. 4A). By contrast, dysplastic acinar cells and acinar cell carcinomas were present in the pancreases of SW 1990 xenograft mice, rather than differentiated acinar cell carcinomas. Large solid tumor masses were also observed in the pancreases of SW 1990 xenograft mice (Fig. 4B). 

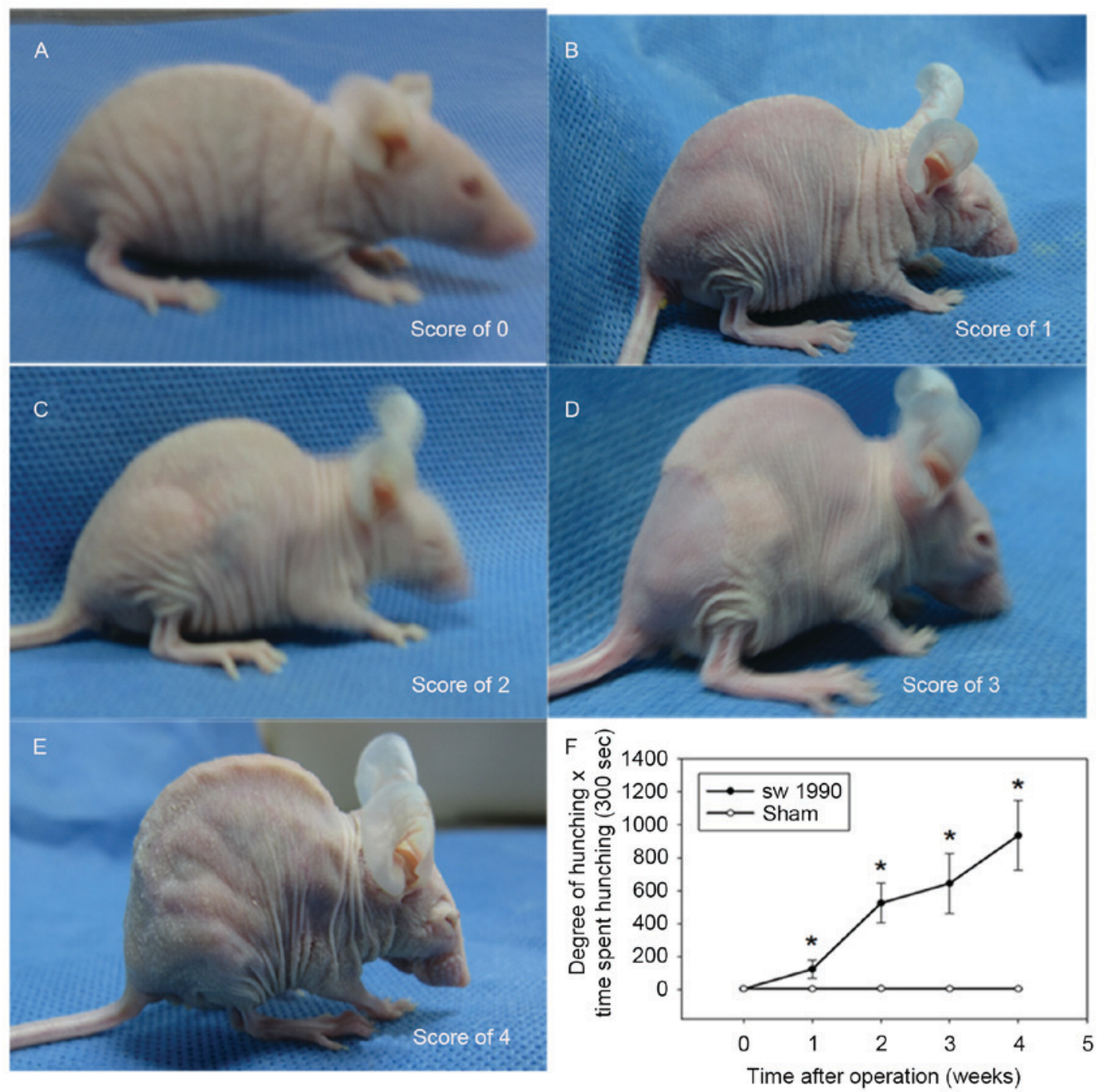

Figure 6. SW 1990 xenografting induces hunching behaviors in mice (n=10, each group). (A-E) Images of mice demonstrating hunching behaviors scored from 0 to 4. (A) 0, Exploratory behavior and lack of a rounded-back posture. (B) 1, Exploratory behavior and mild rounded-back posture. (C) 2, Slightly reduced exploratory behavior, severe rounded-back posture and intermittent abdominal contractions. (D) 3, Markedly reduced exploratory behavior, severe rounded-back posture and intermittent abdominal contractions. (E) 4, Little or no exploratory behavior, severe rounded-back posture and intermittent abdominal contractions. (F) Hunching score based on the accumulated time (sec) engaged in hunching behavior multiplied by the scoring factor associated with the degree of impairment. At week 1 post-SW 1990 xenografting, hunching behavior became evident. The degree of hunching increased continually over time following SW 1990 xenografting. "P<0.001 vs. sham-operated mice post-hoc least significant difference test. SW 1990, SW 1990 cell xenografted mice; Sham, sham-operated mice.

Hypersensitivity to mechanical stimulation in SW 1990 xenografted mice. The hypersensitivity to mechanical stimulation in the abdomen was quantified by the withdrawal threshold, and Fig. 5 presents the behavioral testing results. There was a sharp decline in the mechanical thresholds during weeks 1 and 2 following the SW 1990 xenograft surgery, and the thresholds of the SW 1990 group were significantly reduced compared with the sham group $(\mathrm{P}<0.001$, according to the post hoc LSD test). Notably, the mechanical thresholds in sham-operated mice declined slightly during week 1 , but then became stable when the withdrawal threshold reached the normal level (Fig. 5).

Hunching behaviors in SW 1990 xenografted mice. At week 1 post-SW 1990 xenografting, spontaneous visceral pain-associated behaviors, including hunching, were observed (Fig. 6). The hunching score was based on the degree of hunching and the time spent hunching (Fig. 6A-E). This behavior resembled the 'pancreatic position' assumed by human patients with pancreatic pain (13). As demonstrated in Fig. 6F, the hunching score of SW 1990 xenograft mice was significantly higher compared with the sham-operated mice $(\mathrm{P}<0.001$, according to the post-hoc LSD test). In SW 1990 xenografted mice, spontaneous hunching behaviors became evident on day 8 post-surgery, at which time the hunching score reached 124 . The degree of hunching increased continually over time, and reached 936 on day 28 post-surgery. By contrast, sham-operated mice did not exhibit significant hunching behavior at any timepoint (Fig. 6F).

Increment of VMRs in SW 1990 xenografted mice. VMRs, as a reliable, reproducible measure of visceral nociception (14), were recorded as abdominal muscle electromyographic activity in mice (Fig. 7A). Fig. 7 presents the raw VMRs from SW 1990 


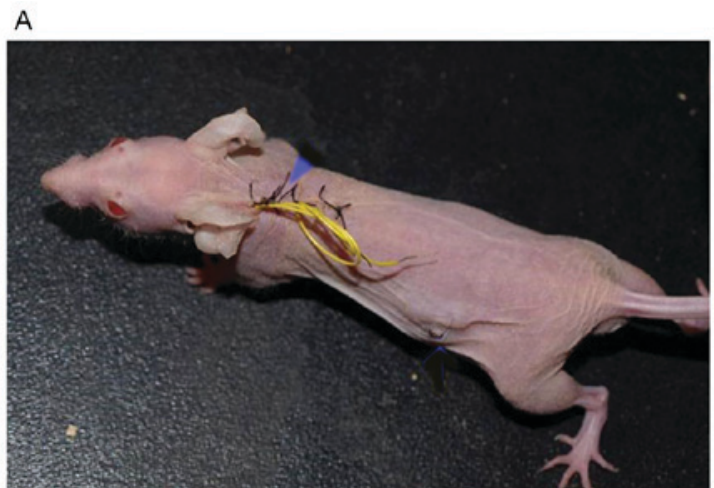

B1

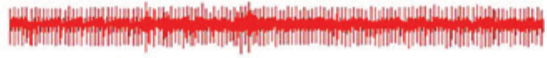

B2
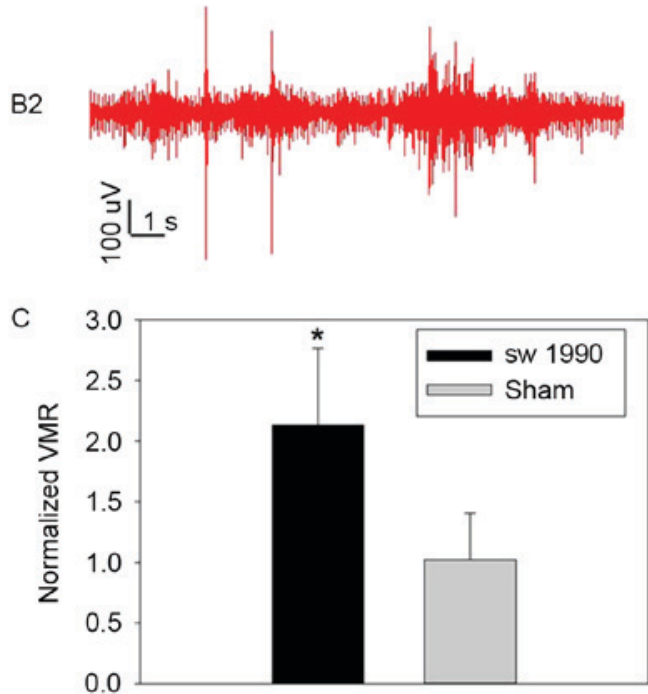

Figure 7. Increment of VMRs in SW 1990 xenografted mice ( $\mathrm{n}=5$ each group). (A) Image of mouse, demonstrating the silver wires implanted in the rectus abdomens for VMR recording. (B1) VMRs of sham-operated mice; the values of the curve were low and regular. (B2) VMRs of SW 1990 xenografted mice; the values of the curve were increased. (C) Normalized VMRs of SW 1990 xenografted mice and sham-operated mice. The VMRs of SW 1990 xenografted mice were significantly higher compared with the sham-operated mice. ${ }^{*} \mathrm{P}<0.001$ vs. sham-operated mice by one-way analysis of variance. SW 1990, SW 1990 cell xenografted mice; Sham, sham-operated mice.

xenografted and sham-operated mice that were graded in relation to the stimulus. Notably, the VMRs in sham-operated mice were low and regular (Fig. 7B1). In comparison, VMRs of the SW 1990 xenografted mice were increased, and the curve was irregular compared with the sham group (Fig. 7B2). Normalized VMRs indicated that the VMRs of SW 1990 xenografted mice were significantly increased compared with the sham group (Fig. 7C).

Genome-wide molecular profiling. To characterize the molecular mechanisms of the pain process, the gene expression profiles of tissue samples from mice with severe cancer pain or with minor pain hypersensitivity were analyzed, using the spinal cord dorsal horn of sham-operated mice as the control. All biological replicates were pooled and calculated to identify differentially expressed genes based on the threshold of fold change and p-value. In total, 26,425 genes expressed in the spinal tissues were detected at sufficient levels for differential expression analysis. The correlation of expression profiles between biological replicates and pain conditions was demonstrated by unsupervised hierarchical clustering analysis, as shown in Fig. 8A. The expression levels of 1,155 genes were upregulated in the severe pain group, whereas 144 genes were demonstrated to be upregulated in the miniature pain group. The expression levels of 1,350 genes were downregulated in the severe pain group, and 92 genes were downregulated in the miniature pain group (Fig. 8A). Comparing the mice with severe and minor cancer pain, 10 genes demonstrated the greatest differences in expression (Fig. 8B). These 10 genes were significantly overexpressed in severe cancer pain mice compared with controls. However, their expression in the minor cancer pain mice were significantly downregulated, or at the basal level, as in sham-operated mice. Of these 10 overexpressed genes, two of them (C112, pin1) have previously been reported in other studies on pain $(14,15)$.

\section{Discussion}

Patients with pancreatic cancer experience severe pain that markedly decreases their quality of life. Certain strategies for pain management are available, including opioid-based pharmacotherapy and non-opioid adjuvant analgesics, such as non-steroidal anti-inflammatory drugs. However, the majority of pain management fails to control pancreatic cancer pain (16-18). Recently, Selvaraj et al (19) used orthotropic mouse models to demonstrate that ligands of the vascular endothelial growth factor family contribute to cancer-associated pain. However, a specific pancreatic cancer-induced pain model is required to mimic reliably the biological behavior of pancreatic cancer in humans, and would represent a useful tool for understanding the mechanisms of pancreatic cancer pain.

The present study demonstrated that orthotropic xenografts of the human SW 1990 cell in BALB/c nu mice successfully established a novel mouse model of pancreatic cancer-induced pain.

The survival rate of pancreatic cancer mice was high during the first 21 days post-surgery and was $>50 \%$ after 4 weeks post-surgery (20). The SW 1990 xenografts also markedly reduced the body weight of the mice. Decreased body weight in patients with cancer is caused by pain and gastric outlet obstruction, leading to a reduced dietary intake. Malabsorption and cachectic syndrome are also involved in weight loss. In a previous study, patients with pancreatic cancer exhibited an elevated resting metabolic rate compared with controls, which may further contribute to the negative energy balance observed in patients with cancer (21). The data of the present study corroborated this result, and gross anatomical analysis demonstrated that the pancreatic tumor volume was increased significantly over time following SW 1990 xenografting, indicating that the mouse model of pancreatic cancer may induce similar metabolic changes.

It is difficult to control pain in patients with pancreatic cancer, as visceral pain does not present until the tumor is highly advanced and quickly metastasizes to other organs (22), Pain caused by pancreatic diseases is often 'referred' to the epigastric area and radiates to the back in humans, as these 

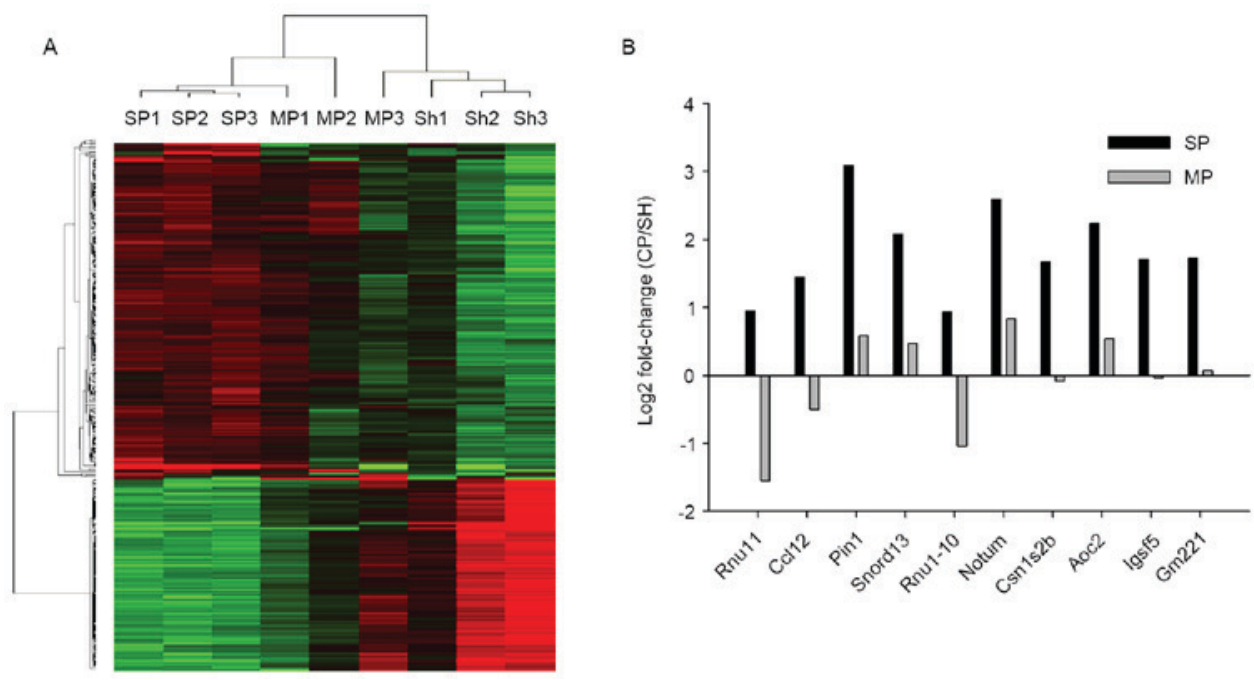

Figure 8. Transcriptome analysis of spinal cord dorsal horn tissue from severe cancer pain, minor cancer pain mouse and sham mice. (A) Evidence for transcriptomic changes in severe cancer pain, minor cancer pain and sham mice. The 9 samples are presented in columns, and the expression pattern (red, upregulation; green, downregulation; black, no change) of the genes is presented in rows. (B) Fold change of the 10 genes that exhibited the greatest differences in expression between severe cancer pain and miniature cancer pain mice. SP, severe cancer pain mice; MP, minor cancer pain mice SH, Sham-operated mice.

dermatomal areas are more susceptible to stimuli (23). Visceral pain leads to a decreased pain threshold following mechanical stimulation of the skin of the abdomen, termed mechanical allodynia (24,25). A similar phenomenon was observed in the animal models of the present study. SW 1990 xenografting continually decreased the mechanical threshold of the abdominal skin in mice, suggesting that visceral pain elicited by pancreatic cancer was aggravated by increased progression of the tumor in the current model.

The transduction pathways and perceptions elicited by visceral pain are different from those of cutaneous pain. The abdominal organs receive dual extrinsic innervation, including spinal and vagal afferents. Somatic pain is precisely localized and characterized, whereas visceral pain is perceived diffusely and is poorly localized. Visceral pain is usually referred to a distant cutaneous or musculoskeletal site (26). Behavioral and autonomic responses to visceral stimulation are observed in patients (27) and animal models (28). In the present study, the mouse model of pancreatic cancer-induced pain caused significant hunching behavior and muscle hypersensitivity in mice.

Previous studies have begun to provide insights into the mechanisms of pancreatic cancer pain. However, clinical practice has yet to be altered by these theoretical advances (29). Genetic mutant mouse models of pancreatic cancer pain remain different from the human disease. However, the mouse model used in the present study exhibits several advantages, including ease of development, high success rate and low mortality, and is considered as a useful animal model. Furthermore, the model produces various pain behaviors that are also observed in human disease.

It was reported recently that a genetic biomarker may predict nerve pain side-effects associated with prostate cancer treatment (30). To understand better which genes are linked to cancer pain may lead to improved pain management for cancer patients who suffer intense and consistent pain, and the present study demonstrated dysregulated gene expression in the spinal cord dorsal horn. The ccl12 gene encoding the protein of chemokine (C-C motif) ligand 12 is involved in cytokine-mediated signaling, mitogen-activated protein kinase cascades, protein kinase B signaling and the initiation of inflammatory responses, which are involved in the mechanisms of pain $(31,32)$. Quick et al $(33)$ identified that CCL12 was upregulated in experimental autoimmune prostatitis by 12-13 fold on day 5, and by 20-35 fold on day 30, and therefore it may be an essential mediator of pelvic pain. The pin1 gene encoding peptidyl-prolyl cis/trans isomerase acts as a negative regulator of the extracellular signal-regulated kinase (ERK) cascade and a positive regulator of protein phosphorylation and cytokinesis. Guan et al (34) have demonstrated Akt-ERK crosstalk mediates bone cancer pain. It was also reported that pin1 regulates cadmium-induced autophagy via ubiquitin-mediated post-translational stabilization of glycogen synthase kinase 3 (GSK3) $\beta$ and inhibition of GSK3 $\beta$ signaling, resulting in enhanced dorsal root ganglia growth responses, and that PI3K-mediated activation of GSK-3 $\beta$ is able to prevent this growth and the development of at-level pain syndromes (35). The notum gene encoding palmitoleoyl-protein carboxylesterase is involved in Wnt signaling, which is critical for the induction and maintenance of chronic neuropathic pain (36). The present results demonstrated that ccl12, pin1 and notum were overexpressed genes in the pain model, which is consistent with these studies (Fig. 8). In addition, increased expression levels of the rnu11, snord13 and rnu1-10 genes were also observed; these code for small nuclear RNAs and the genes csn1s $2 b$, aoc, igsc 5 and gm221, but their expression may not be directly involved in the current model of pain transmission. The clinical relevance of these findings remains to be elucidated in further studies. The expression levels of the genes identified within the microarray experiment were not examined further by quantitative real time polymerase chain reaction; however, the results demonstrated a trend.

In conclusion, the present study developed a novel animal model of pancreatic cancer pain in BALB/c-nu mice. The 
model enabled the identification of several pancreatic cancer pain-associated genes that were differentially expressed in the spinal cord dorsal horn. This model may potentially be used as an improved in vivo tool for preclinical studies of pancreatic cancer pain, and to provide further insights into the pathogenic processes of cancer pain.

\section{Acknowledgements}

This work was supported by the National Natural Science Foundation of China (grant nos. 31171013 and 31371084 to Z.L. and 81200335 to Qianbo Chen) and the Braun Fund for Anesthesia Scientific Research (grant no. BBF2011-007 to Z.L.).

\section{References}

1. Mantyh PW: Cancer pain and its impact on diagnosis, survival and quality of life. Nat Rev Neurosci 7: 797-809, 2006.

2. Van den Beuken-van Everdingen MH, de Rijke JM, Kessels AG, Schouten HC, van Kleef M and Patijn J: Prevalence of pain in patients with cancer: A systematic review of the past 40 years. Ann Oncol 18: 1437-1449, 2007.

3. Breivik H, Cherny N, Collett B, de Conno F, Filbet M, Foubert AJ, Cohen R and Dow L: Cancer-related pain: A pan-European survey of prevalence, treatment, and patient attitudes. Ann Oncol 20: 1420-1433, 2009 .

4. Gupta M, Sahi MS, Bhargava AK and Talwar V: The prevalence and characteristics of pain in critically III cancer patients: A prospective nonrandomized observational study. Indian J Palliat Care 21: 262-267, 2015.

5. Sindt JE and Brogan SE: Interventional treatments of cancer pain. Anesthesiol Clin 34: 317-339, 2016.

6. Deer TR, Smith HS, Burton AW, Pope JE, Doleys DM, Levy RM, Staats PS, Wallace MS, Webster LR, Rauck RL, et al Comprehensive consensus based guidelines on intrathecal drug delivery systems in the treatment of pain caused by cancer pain Pain Physician 14: E283-E312, 2011.

7. Fillingim RB, Bruehl S, Dworkin RH, Dworkin SF, Loeser JD, Turk DC, Widerstrom-Noga E, Arnold L, Bennett R, Edwards RR, et al: The ACTTION-American Pain Society Pain Taxonomy (AAPT): An evidence-based and multidimensional approach to classifying chronic pain conditions. J Pain 15 241-249, 2014.

8. Tevethia MJ, Bonneau RH, Griffith JW and Mylin L: A simian virus 40 large $\mathrm{T}$-antigen segment containing amino acids 1 to 127 and expressed under the control of the rat elastase- 1 promoter produces pancreatic acinar carcinomas in transgenic mice. J Virol 71: 8157-8166, 1997.

9. Loukopoulos P, Kanetaka K, Takamura M, Shibata T, Sakamoto $\mathrm{M}$ and Hirohashi S: Orthotopic transplantation models of pancreatic adenocarcinoma derived from cell lines and primary tumors and displaying varying metastatic activity. Pancreas 29: 193-203, 2004.

10. Sevcik MA, Jonas BM, Lindsay TH, Halvorson KG, Ghilardi JR, Kuskowski MA, Mukherjee P, Maggio JE and Mantyh PW: Endogenous opioids inhibit early-stage pancreatic pain in a mouse model of pancreatic cancer. Gastroenterology 131 900-910, 2006

11. Yan XJ, Feng CC, Liu Q, Zhang LY, Dong X, Liu ZL, Cao ZJ, Mo JZ, Li Y, Fang JY and Chen SL: Vagal afferents mediate antinociception of estrogen in a rat model of visceral pain: The involvement of intestinal mucosal mast cells and 5-hydroxytryptamine 3 signaling. J Pain 15: 204-217, 2014.

12. Cao DY, Ji Y, Tang B and Traub RJ: Estrogen receptor $\beta$ activation is antinociceptive in a model of visceral pain in the rat. J Pain 13: 685-694, 2012.

13. Pitchumoni CS: Pathogenesis and managenent of pain in chronic pancreatitis. World J Gastroenterol 6: 490-496, 2000.

14. Poh KW, Yeo JF, Stohler CS and Ong WY: Comprehensive gene expression profiling in the prefrontal cortex links immune activation and neutrophil infiltration to antinociception. J Neurosci 32 $35-45,2012$.
15. Yu L, Wu X, Wei J, Liao Q, Xu L, Luo S, Zeng X, Zhao Y, Lv Z and Wu Z: Preliminary expression profile of cytokines in brain tissue of BALB/c mice with Angiostrongylus cantonensis infection. Parasit Vectors 8: 328, 2015.

16. Portenoy RK: Treatment of cancer pain. Lancet 377: 2236-2247, 2011.

17. Forman AD: Peripheral neuropathy and cancer. Curr Oncol Rep 6: 20-25, 2004.

18. Cherny NI: How to deal with difficult pain problems. Ann Oncol 16 (Suppl 2): ii79-ii87, 2005.

19. Selvaraj D, Gangadharan V, Michalski CW, Kurejova M, Stösser S, Srivastava K, Schweizerhof M, Waltenberger J, Ferrara N, Heppenstall P, et al: A functional role for VEGFR1 expressed in peripheral sensory neurons in cancer pain. Cancer Cell 27: 780-796, 2015.

20. Ness TJ and Elhefni H: Reliable visceromotor responses are evoked by noxious bladder distention in mice. J Urol 171: 1704-1708, 2004

21. Argilés JM, Busquets S, Garcia-Martinez C and López-SorianoFJ: Mediators involved in the cancer anorexia-cachexia syndrome: Past, present, and future. Nutrition 21: 977-985, 2005.

22. Wigmore SJ, Plester CE, Richardson RA and Fearon KC: Changes in nutritional status associated with unresectable pancreatic cancer. Br J Cancer 75: 106-109, 1997.

23. Kelsen DP, Portenoy R, Thaler H, Tao Y and Brennan M: Pain as a predictor of outcome in patients with operable pancreatic carcinoma. Surgery 122: 53-59, 1997.

24. Vera-Portocarrero LP, Lu Y and Westlund KN: Nociception in persistent pancreatitis in rats: Effects of morphine and neuropeptide alterations. Anesthesiology 98: 474-484, 2003.

25. Laird JM, Olivar T, Roza C, De Felipe C, Hunt SP and Cervero F: Deficits in visceral pain and hyperalgesia of mice with a disruption of the tachykinin NK1 receptor gene. Neuroscience 98: 345-352, 2000.

26. Brink TS and Mason P: Role for raphe magnus neuronal responses in the behavioral reactions to colorectal distension. J Neurophysiol 92: 2302-2311, 2004.

27. Giamberardino MA, de Bigontina P, Martegiani $\mathrm{C}$ and Vecchiet L: Effects of extracorporeal shock-wave lithotripsy on referred hyperalgesia from renal/ureteral calculosis. Pain 56: 77-83, 1994.

28. Giamberardino MA, Valente R, de Bigontina P and Vecchiet L: Artificial ureteral calculosis in rats: Behavioural characterization of visceral pain episodes and their relationship with referred lumbar muscle hyperalgesia. Pain 61: 459-469, 1995.

29. Gregory NS, Harris AL, Robinson CR, Dougherty PM, Fuchs PN and Sluka KA: An overview of animal models of pain: Disease models and outcome measures. J Pain 14: 1255-1269, 2013.

30. Magrangeas F, Kuiper R, Avet-Loiseau H, Gouraud W, Guérin-Charbonnel C, Ferrer L, Aussem A, Elghazel H, Suhard J, Sakissian H, et al: A genome-wide association study identifies a novel locus for bortezomib-induced peripheral neuropathy in European patients with multiple myeloma. Clin Cancer Res 22: 4350-4355, 2016

31. Li W, Zhang Y, Xing $\mathrm{C}$ and Zhang $\mathrm{M}$ : Tanshinone IIA represses inflammatory response and reduces radiculopathic pain by inhibiting IRAK-1 and NF- $\mathrm{KB} / \mathrm{p} 38 / \mathrm{JNK}$ signaling. Int Immunopharmacol 28: 382-389, 2015.

32. Li Y, Zhang H, Kosturakis AK, Cassidy RM, Zhang H, Kennamer-Chapman RM, Jawad AB, Colomand CM, Harrison DS and Dougherty PM: MAPK signaling downstream to TLR4 contributes to paclitaxel-induced peripheral neuropathy. Brain Behav Immun 49: 255-266, 2015.

33. Quick ML, Mukherjee S, Rudick CN, Done JD, Schaeffer AJ and Thumbikat P: CCL2 and CCL3 are essential mediators of pelvic pain in experimental autoimmune prostatitis. Am J Physiol Regul Integr Comp Physiol 303: R580-R589, 2012.

34. Guan XH, Fu QC, Shi D, Bu HL, Song ZP, Xiong BR, Shu B, Xiang HB, Xu B, Manyande A, et al: Activation of spinal chemokine receptor CXCR3 mediates bone cancer pain through an Akt-ERK crosstalk pathway in rats. Exp Neurol 263: 39-49, 2015.

35. Bareiss SK, Dugan E and Brewer KL: PI3K mediated activation of GSK-3 $\beta$ reduces at-level primary afferent growth responses associated with excitotoxic spinal cord injury dysesthesias. Mol Pain 11: 35, 2015 .

36. Feng W, Teng R, Zhao Y, Gao J and Chu H: Epigenetic modulation of Wnt signaling contributes to neuropathic pain in rats. Mol Med Rep 12: 4727-4733, 2015. 